\title{
EVALUATION OF THE DEDEKIND ETA FUNCTION
}

\author{
ROBIN CHAPMAN AND WILLIAM HART
}

\begin{abstract}
We extend the methods of Van der Poorten and Chapman [7] for explicitly evaluating the Dedekind eta function at quadratic irrationalities. Via Hecke $L$-series we obtain evaluations in some new cases. Specifically we provide further evaluations at points in imaginary quadratic number fields with class numbers up to four. We also describe techniques, which make use of modular equations, which provide additional evaluations not obtained via the $L$-series techniques, and we give a number of these evaluations explicitly here.
\end{abstract}

\section{INTRODUCTION}

The Dedekind eta function is defined for $\tau$ on the upper half complex plane by the $q$-series

$$
\eta(\tau)=q^{1 / 24} \prod_{n=1}^{\infty}\left(1-q^{n}\right)
$$

where $q=e^{2 \pi i \tau}$. It is a 24-th root of the discriminant function $\Delta(\tau)$ of an elliptic curve.

In the literature evaluations of eta, at points in imaginary quadratic number fields, apparently go back to Abel [1] who showed that $\eta(\sqrt{-5} / 2) / 2 \eta^{2}(2 \sqrt{-5})$ is a root of the palindromic quartic $x^{4}-2 x^{3}-2 x^{2}-2 x+1$. For this and some other facts related to the history of the subject see the introduction to [10].

More recently evaluations of quotients of the eta function have received new attention due to the fact that they provide explicit generators of ring class fields (see $[12])$. These are of some importance to cryptography and computer primality tests (see $[6]$ ).

It was Chowla and Selberg in 1949 who announced the first formula for eta evaluations. Their forthcoming paper [9] outlined a method that relied on evaluating sums of the form

$$
Z(s)=\sum^{\prime}\left(a x^{2}+b x y+c y^{2}\right)^{-s}
$$

so-called Epstein zeta functions. This led to the following formula, a slight rearrangement of formula (2) on p. 110 of [9], which has been called the ChowlaSelberg formula

$$
\prod_{j=1}^{h} a_{j}^{-6} \Delta\left(\tau_{j}\right)=(2 \pi|d|)^{-6 h}\left[\prod_{m=1}^{|d|} \Gamma\left(\frac{m}{|d|}\right)^{\left(\frac{d}{m}\right)}\right]^{3 w} .
$$

Here the product on the left is over all the reduced binary quadratic forms $\left(a_{j}, b_{j}, c_{j}\right)$ with negative fundamental discriminant $d$, the class number being $h$, and with $\tau_{j}=\frac{b_{j}+\sqrt{d}}{2 a_{j}}$ a root of the quadratic form $\left(a_{j}, b_{j}, c_{j}\right)$. On the right is essentially a

Date: Preprint 2003. 
product of gamma functions with the exponents $\left(\frac{d}{m}\right)$ given by the Legendre-JacobiKronecker symbol. Also $w$ is the number of roots of unity in the quadratic field $\mathbb{Q}(\sqrt{d})$.

Taking 24-th roots and absolute values on both sides of (3) yields a formula involving a product of absolute values of eta functions.

Clearly if there is only one form in the form class group, the formula provides an evaluation of the modulus of a single eta function in terms of gamma functions.

Through a long series of improvements, the Chowla-Selberg formula has been progressively extended. First Kaneko [19] and Nakajima and Taguchi [20] extended the formula to non-fundamental discriminants. Later Williams and Zhang [3] refined the formula so that the product on the left hand side could be taken over a single genus of reduced forms of fundamental discriminant. Finally Huard, Kaplan and Williams [8] did the same thing for non-fundamental discriminant.

These formulae appear to have been inspired in part by Zucker and Robertson who have a series of papers [21], [22], [23], [24] on their technique of 'solving' $L$-series, i.e. they split various $L$-series into quadratic $L$-series and use the formulae of Dirichlet to provide explicit evaluations of them.

However there still existed no method to evaluate an isolated eta function corresponding to the root of a single reduced form, except in the cases where there was a single form per genus.

More recently Williams and van der Poorten [18] produced a formula breaking up the Chowla-Selberg formula completely. Later Chapman and van der Poorten [7] recognized various quantities to be generalised class group characters which led to the expression of eta evaluations in terms of Hecke L-series. Evaluating various of these L-series meant that various explicit evaluations of isolated eta functions could then be given. In particular, a neat trick allowed the evaluation of a single eta function at a point in a quadratic field of fundamental discriminant $d=-31$, whose class number is 3 .

Evaluations are also provided in [7] where the class number is three and the discriminant $D$ is non-fundamental. However, in that analysis, one relies on the fact that the class number is the same in the order of discriminant $D$ as it is in the maximal order (the ring of integers) of the underlying quadratic number field.

Nonetheless an analysis of techniques prior to these revealed that complete evaluations had generally only been done in cases where a genus character would suffice to separate the quadratic forms involved. This corresponds to cases where the extended class group of the quadratic order, from which our quadratic values are taken, is 2-torsion.

In the present paper we show how more general results can be obtained which do not have the restrictions mentioned above. We also provide a number of explicit evaluations in these new situations. Our hope is that these ideas may eventually lead to a more general theory.

In $\S 1$ we describe an explicit eta evaluation where the discriminant $(D=-44)$ is non-fundamental but the class number of the order of discriminant $D$ is not the same as that of the maximal order of the underlying quadratic number field.

$\S 2$ uses minimal computational assistance to deal with fundamental discriminant and class number four $(d=-56)$.

In $\S 3$ we report on a solution for the case of fundamental discriminant and class number five $(d=-47)$, using a known evaluation of a Weber function.

In $\S 4$ we use the results of [14] on modular equations for new Weber-like functions to provide an evaluation where the class number is seven. 


\section{Evaluation of Eta for Non-fundamental Discriminant $D=-44$}

Our first eta evaluations will be at roots of binary quadratic forms with discriminant $D=-44$. This is a non-fundamental discriminant of an order with class number three. The underlying quadratic number field has discriminant $d=-11$ and class number 1 . This is a situation not dealt with by the methods of [7]. However a simple modification of those techniques suffices to deal with this case.

The primitive reduced forms for $D=-44$ are $Q_{1}=(1,0,11), Q_{2}=(3,2,4)$ and $Q_{3}=(3,-2,4)$. The sole reduced form of discriminant $d=-11$ is $Q^{\prime}=(1,1,3)$.

The generalised Chowla-Selberg formula ([19], [20]) for the non-fundamental discriminant $D=-44$ therefore gives us the product

$$
3^{-\frac{1}{2}}\left|\eta(\sqrt{-11}) \eta\left(\frac{1+\sqrt{-11}}{3}\right) \eta\left(\frac{-1+\sqrt{-11}}{3}\right)\right|=\left(\frac{1}{88 \pi}\right)^{\frac{3}{4}} \Gamma\left(\frac{\cdot}{11}\right)^{\frac{3}{4}} 2^{\frac{1}{2}}
$$

Here we have used the abbreviation

$$
\Gamma\left(\frac{\dot{z}}{d}\right)=\prod_{n=1}^{d} \Gamma\left(\frac{n}{|d|}\right)^{\left(\frac{d}{n}\right)}
$$

where $\left(\frac{d}{n}\right)$ is the Legendre-Jacobi-Kronecker symbol and $\Gamma\left(\frac{n}{d}\right)$ is the classical gamma function.

Now that we have the product of all the eta functions in question, it will suffice to determine pairwise quotients of them in order to find all their separate values.

Notice first of all, from the definition of $\eta(\tau)$, that

$$
\left|\eta\left(\frac{b+\sqrt{D}}{2 a}\right)\right|=\left|\eta\left(\frac{-b+\sqrt{D}}{2 a}\right)\right|
$$

thus the absolute values of the last two eta functions in (4) are equal, so all that remains is to find the absolute value of the quotient of the first two eta values.

In order to evaluate the required quotient we proceed in a manner which is similar to [7]. Letting $\omega$ represent the cube root $e^{2 \pi i / 3}$ we evaluate the sum $S(s, k)$

$$
\begin{array}{r}
\sum^{\prime} \frac{1}{\left(x^{2}+11 y^{2}\right)^{s}}+\sum^{\prime} \frac{\omega^{k}}{\left(3 x^{2}+2 x y+4 y^{2}\right)^{s}}+\sum^{\prime} \frac{\omega^{2 k}}{\left(3 x^{2}-2 x y+4 y^{2}\right)^{s}} \\
=\sum_{m=1}^{\infty} \frac{N\left(Q_{1}, m\right)}{m^{s}}+\omega^{k} \sum_{m=1}^{\infty} \frac{N\left(Q_{2}, m\right)}{m^{s}}+\omega^{2 k} \sum_{m=1}^{\infty} \frac{N\left(Q_{3}, m\right)}{m^{s}}
\end{array}
$$

where $N\left(Q_{i}, m\right)$ is the number of representations of the integer $m$ by the form $Q_{i}$, and where the dashes indicate that we sum only over pairs of integers $(x, y)$ which are not both zero.

Now we use the formula

$$
N(Q, m)=\sum_{r \mid \kappa} N_{R}\left(Q_{r}, m / r^{2}\right)
$$

from section 2.4 of [7]. Here $N_{R}(Q, m)$ represents the number of regular representations of $m$ by the form $Q$, i.e. where $m$ is coprime to the conductor of $Q$. It is defined to be zero when $m$ is not an integer. Note also that the notation in this formula is different to ours. The form $Q$ in this equation corresponds to any one of the three forms of discriminant $D$ which we have mentioned above, whilst the form $Q_{r}$ is of discriminant $D / r^{2}$ and is obtained from $Q$ by a canonical process described in [7] sections 2.1 and 2.2 . 
In our case the conductor $\kappa=2$, and all three forms of discriminant $D=-44$ drop down to the single form of discriminant $d=-11$ when $r=2$ in the sum. When $r=1$, the three forms remain unchanged. Thus using this formula our sum, appearing above, can be rewritten as

$$
\begin{array}{r}
S(s, k)=\sum_{n=1}^{\infty} \frac{N_{R}\left(Q_{1}, m\right)}{m^{s}}+\omega^{k} \sum_{n=1}^{\infty} \frac{N_{R}\left(Q_{2}, m\right)}{m^{s}}+\omega^{2 k} \sum_{n=1}^{\infty} \frac{N_{R}\left(Q_{3}, m\right)}{m^{s}} \\
\quad+\left(1+\omega^{k}+\omega^{2 k}\right) \sum_{n=1}^{\infty} \frac{N_{R}\left(Q^{\prime}, m / 4\right)}{m^{s}}
\end{array}
$$

where the quadratic forms are now given in our original notation. Note that for $k=1,2$ the fourth term of this expression is zero. These cases will suffice for our purposes.

So analogously to equation (1) of [7] the sum becomes simply

$$
S(s, k)=2 \sum_{\substack{\mathfrak{a} \in I_{2} \\ \text { integral }}} \frac{\chi[\mathfrak{a}]}{(N \mathfrak{a})^{s}}
$$

where the character $\chi$ is non-trivial and depends on whether $k=1$ or 2 , the sum is over all integral ideals of $\mathcal{O}_{K}$ which are coprime to 2 and the factor of 2 that appears is the number of roots of unity in the order of discriminant $D=-44$. Note that the other expressions $r$ and $g(\kappa ; r)$ that appear in (1) of [7] have the value 1 in this situation.

Now we come to the departure from the methods of [7]. We view the sum on the right hand side of $(10)$ as a primitive $L$-series of a ring class extension of our underlying quadratic field $K$ of discriminant $d=-11$. We think of the conductor of our order of discriminant $D=-44$ as a modulus of a class field extension $R$ of $K$, which is called the ring class field of conductor 2 of $K$.

We let $G=G(R / K)$ be the Galois group of the ring class extension. Consider the character group $\hat{G}$ of $G$. Then our equation (10) can be written

$$
S(s, \chi)=2 L(s, \chi)=\prod_{\mathfrak{p}}\left(1-\frac{\chi(\mathfrak{p})}{(N \mathfrak{p})^{s}}\right)^{-1}
$$

in terms of a primitive $L$-series for a character $\chi$ taken over all the prime ideals of $K$ in a way which we will make precise.

We view our character $\chi$ defined on prime ideals here as coming from some appropriate $\chi \in \hat{G}$ which has been extended to a character on ideals via the Frobenius automorphisms $\sigma_{\mathfrak{p}}$ as follows. If $\chi \in \hat{G}$ vanishes on the inertia group of $\mathfrak{p}$ then $\chi(\mathfrak{p})$ is set to equal $\chi\left(\sigma_{\mathfrak{p}}\right)$, otherwise $\chi(\mathfrak{p})$ is set to zero.

In our very simple case here, this formulation simply guarantees that $\chi$ vanishes on all prime ideals of $K$ which divide 2 . At any rate, the $L$-series we are after is one of the primitive $L$-series corresponding to the ring class field extension we have mentioned.

Now taking the limit as $s \rightarrow 1$ in (11) and applying the Kronecker limit formula to the same expression $S(s, k)=S(s, \chi)$, but as given by (7), we obtain

$$
-\frac{8 \pi}{\sqrt{44}} \log \left|\frac{\eta(\sqrt{-11})}{3^{-\frac{1}{4}} \eta\left(\frac{1+\sqrt{-11}}{3}\right)}\right|=2 L(1, \chi) .
$$


It remains only to calculate $L(1, \chi)$ for which we make use of the expression

$$
\zeta_{R}(s)=\prod_{\chi \in \hat{G}} L(s, \chi)
$$

expressing the Dedekind zeta function of the ring class field $R$ in terms of the primitive $L$-series of the extension $R / K$, one of which is the Dedekind zeta function of $K$. We are now in a situation analogous to the calculation of $L(1, \chi)$ that arose for the discriminant $d=-31$ in [7].

In particular $L(1, \chi)=L\left(1, \chi^{2}\right)$ and the $L$-series we are after is given by

$$
L(1, \chi)^{2}=\lim _{s \rightarrow 1^{+}} \frac{\zeta_{R}(s)}{\zeta_{K}(s)}
$$

the quotient of the zeta functions.

Again by the analytic class number formula

$$
\lim _{s \rightarrow 1^{+}}(s-1) \zeta_{F}(s)=\frac{2^{s+t} \pi^{t} h_{F} R_{F}}{W_{F} \sqrt{\left|d_{F}\right|}}
$$

where $s$ and $2 t$ are the numbers of real and complex embeddings of $F, h_{F}$ is the class number, $R_{F}$ is the regulator, $W_{f}$ is the number of roots of unity and $d_{F}$ is the discriminant of $F$.

Now in our case, Cox [5], for example, asserts that the ring class field $R$ is generated over $K$ by the square of the Weber function $x=f(\sqrt{-11})$. It is known from the tables at the end of Weber's book [17] that $x$ satisfies $x^{3}-2 x^{2}+2 x-2=0$. Letting $u=x^{2}$ we see easily that $u$ satisfies $u^{3}-4 u-4=0$. Pari then easily calculates that the discriminant of $R / Q$ is $-2^{4} 11^{3}$, the class number is 1 and of course the number of roots of unity is 2 .

Letting $R_{R}$ denote the regulator of the field $R$, we obtain the expression

$$
L(1, \chi)=\frac{\pi}{\sqrt{11}} \sqrt{R_{R}}
$$

Combining this with our expression (12) we finally obtain

$$
\left|\frac{\eta(\sqrt{-11})}{\eta\left(\frac{1+\sqrt{-11}}{3}\right)}\right|=3^{-1 / 4} e^{-\sqrt{R_{R}} / 2} .
$$

We note that with a little care one can do all of the above with respect to the relative extension $R / K$, or what is essentially the same thing, with respect to the real subfield $N$ of $R$ which is generated over $\mathbb{Q}$ by the real root of $u^{3}-4 u-4$. This field has a single fundamental unit $v$ and its regulator is just the logarithm of the absolute value of this unit. In fact

$$
\left|\frac{\eta\left(\frac{1+\sqrt{-11}}{3}\right)}{\eta(\sqrt{-11})}\right|^{2}=\sqrt{3} v
$$

\section{Evaluation of Eta FOR $d=-56$.}

At first sight it would appear that the technique of the previous section, where an $L$-series is expressed in terms of a quotient of zeta functions, can only be successful when the class number of the quadratic order under consideration is three. In this section we see that similar techniques can however be applied in more general situations. 
In particular, in this section we consider a case with fundamental discriminant $d=-56$, but where the class number is four. In fact there are four reduced binary quadratic forms for this discriminant, $(1,0,14),(2,0,7),(3,2,5)$ and $(3,-2,5)$.

Again using the techniques of [7] we have that

$$
\begin{aligned}
\sum^{\prime} \frac{1}{\left(x^{2}+47 y^{2}\right)^{s}} & +\sum^{\prime} \frac{i^{k}}{\left(3 x^{2}+2 x y+5 y^{2}\right)^{s}} \\
& +\sum^{\prime} \frac{i^{2 k}}{\left(2 x^{2}+7 y^{2}\right)^{s}}+\sum^{\prime} \frac{i^{3 k}}{\left(3 x^{2}-2 x y+5 y^{2}\right)^{s}}=2 L(s, \chi)
\end{aligned}
$$

where again the $L$-series and its character $\chi$ belong to some extension of the underlying quadratic number field of discriminant $d=-56$.

By class field theory this extension field will be the Hilbert class field $H$ of the underlying quadratic number field $K$. The Hilbert Class Field $H$ in this case is a cyclic extension of degree four of the quadratic number field $K=\mathbb{Q}(\sqrt{-14})$.

The Hilbert class field can also be viewed as a quadratic extension of an intermediate field $L$ which is itself a quadratic extension of $K$. Let the Galois group of $H / K$ be generated by $\sigma$. Then we can take $L$ to be the intermediate field which is the fixed field of $\sigma^{2}$. This field $L$ also plays an important part in what follows.

In particular for these fields, if $\chi$ is a generator of the character group of $G=$ $\operatorname{Gal}(H / K)$, we have the relations

$$
\begin{gathered}
\zeta_{H}(s)=\zeta_{K}(s) L(s, \chi) L\left(s, \chi^{2}\right) L\left(s, \chi^{3}\right) \\
\zeta_{L}(s)=\zeta_{K}(s) L\left(s, \chi^{2}\right)
\end{gathered}
$$

between the various primitive $L$-series of the extensions $H / K$ and $L / K$ respectively. We note that $L\left(s, \chi^{2}\right)$ is the same $L$-series in both of these expressions.

Note also that $L(s, \chi)=L(s, \bar{\chi})=L\left(s, \chi^{3}\right)$. It is now clear that $L\left(s, \chi^{2}\right)$ can be calculated from the second of these relations, then allowing $L(s, \chi)$ to be calculated from the first, i.e. if $\xi(F)$ denotes the residue at $s=1$ of the zeta function $\zeta_{F}(s)$ of the number field $F$, we have that

$$
L\left(1, \chi^{2}\right)=\frac{\xi(L)}{\xi(K)} \quad \text { and } \quad L(1, \chi)=\sqrt{\frac{\xi(H)}{\xi(L)}} .
$$

The fields $K, L$ and $H$ have class numbers 4,2 and 1 and discriminants $-56,56^{2}$ and $56^{4}$ respectively. All the fields contain 2 roots of unity. Applying the formula (15), with these values, then allows us to obtain expressions for the $L$-series in terms of the regulators of the fields $H$ and $L$.

We can apply the Kronecker limit formula to the left hand side of (19) and thus it is possible to derive expressions for the logarithms of quotients of the various eta values in terms of the regulators of $L$ and $H$ in a similar manner to the previous section.

This method however leads to some undesirable quantities appearing in the final solution. In particular, the regulator of the field $H$ is not the logarithm of a single fundamental unit. In order to improve the situation, we make use of Pari [11] to manipulate the units involved. The result turns out to be much neater, but of course relies on the additional information we are able to gain numerically.

We let $\alpha=\sqrt{-1+2 \sqrt{2}}$ and $\beta=\sqrt{-1-2 \sqrt{2}}$. We note that $\alpha \beta=\sqrt{-7}$. The Hilbert class field of $K$ is in fact $H=\mathbb{Q}(\sqrt{-14}, \alpha)=\mathbb{Q}(\sqrt{-14}, \beta)=\mathbb{Q}(\alpha, \beta)$. 
The Galois group of $H / \mathbb{Q}$ is dihedral of order 8 . It is generated by $\sigma$ of order 4 whose action is characterised by

$$
\sigma(\alpha)=\beta, \sigma(\beta)=-\alpha,
$$

and by complex conjugation denoted $\tau$.

With these definitions we were able to find with Pari, the following set of units of $H$

$$
\begin{aligned}
& \epsilon_{0}=(1+\alpha+\alpha \sqrt{2}) / 2, \quad \epsilon_{1}=(1+\beta-\beta \sqrt{2}) / 2 \\
& \epsilon_{2}=(1-\alpha-\alpha \sqrt{2}) / 2 \quad \epsilon_{3}=(1-\beta+\beta \sqrt{2}) / 2,
\end{aligned}
$$

any three of which constitute a system of fundamental units of $H$.

They are all roots of

$$
\lambda^{4}-2 \lambda^{3}-\lambda^{2}+2 \lambda-1=0,
$$

and they have the property that $\sigma\left(\epsilon_{n}\right)=\epsilon_{n+1}$ and $\tau\left(\epsilon_{n}\right)=\epsilon_{-n}$ with subscripts modulo 4.

Now we let $l_{i}$ be the logarithm of the absolute value of the unit $\epsilon_{i}$. In this notation, $l_{0}+l_{1}+l_{2}+l_{3}=0$ and since $\epsilon_{1}$ and $\epsilon_{3}$ are non-real complex conjugates, we have that $l_{1}=l_{3}=-\frac{1}{2}\left(l_{0}+l_{2}\right)$. Also the regulator of $L$ turns out to be $R_{K}=2\left(l_{0}+l_{2}\right)$ since the fundamental unit of $L$ is given by $1+\sqrt{2}=\left|\epsilon_{0} \epsilon_{2}\right|$.

The regulator of $H$ can now be written as the following determinant

$$
\left|\begin{array}{ccc}
2 l_{0} & -\left(l_{0}+l_{2}\right) & 2 l_{2} \\
-\left(l_{0}+l_{2}\right) & 2 l_{0} & -\left(l_{0}+l_{2}\right) \\
2 l_{2} & -\left(l_{0}+l_{2}\right) & 2 l_{0}
\end{array}\right|=4\left(l_{0}+l_{2}\right)\left(l_{0}-l_{2}\right)^{2} .
$$

Using the analytic class number formula we can now express the residues (after simplification) as

$$
\xi(K)=\frac{2 \pi}{\sqrt{14}}, \quad \xi(L)=\frac{\pi^{2}\left(l_{0}+l_{2}\right)}{7}, \quad \xi(H)=\frac{\pi^{4}\left(l_{0}+l_{2}\right)\left(l_{0}-l_{2}\right)^{2}}{2 \cdot 7^{2}} .
$$

Now we can retrieve the values of the L-series as per the relations above

$$
L\left(1, \chi^{2}\right)=\frac{\pi\left(l_{0}+l_{2}\right)}{\sqrt{14}}, \quad \text { and } \quad L(1, \chi)=\frac{\pi\left(l_{0}-l_{2}\right)}{\sqrt{14}} .
$$

Finally, by applying the Kronecker limit formula to the Epstein zeta functions of (19), we have

$$
\log |\eta(\sqrt{-14})|+\log \left|2^{-\frac{1}{4}} \eta\left(\frac{\sqrt{-14}}{2}\right)\right|-2 \log \left|3^{-\frac{1}{4}} \eta\left(\frac{1+\sqrt{-14}}{3}\right)\right|=-\frac{1}{2}\left(l_{0}+l_{2}\right)
$$

and

$$
\log |\eta(\sqrt{-14})|-\log \left|2^{-\frac{1}{4}} \eta\left(\frac{\sqrt{-14}}{2}\right)\right|=-\frac{1}{2}\left(l_{0}-l_{2}\right) .
$$

It is now clear that by adding or subtracting these two equations, one has expressions for the quotients of eta values. Combining this with the information provided by the Chowla-Selberg formula then leads to complete evaluations of all the eta values involved.

This computation raises the possibility that, in some cases at least, a more direct method may exist which relates eta quotients to some canonical choice of fundamental units. 


\section{Evaluation of Eta FOR $d=-47$.}

In this section we will provide an evaluation of an eta quotient at a point in a quadratic number field, of discriminant $d=-47$, which has class number five. We will not provide eta evaluations for all roots of reduced forms of this discriminant but will provide solely an evaluation of the quotient

$$
a=\left|\frac{\eta((1+\sqrt{-2}) / 4)}{\eta((1+\sqrt{-2}) / 2)}\right| .
$$

Further evaluations for the discriminant $d=-47$ have been completed and will be reported in the thesis [13] of the second author. This simple, but nevertheless interesting, eta quotient is evaluated here primarily to motivate the material of the next section.

We begin by noticing that the eta quotient above can be expressed in terms of one of the Weber functions (see [17] $§ 34$ )

$$
\mathfrak{f}(\tau)=e^{-\frac{\pi i}{24}} \frac{\eta\left(\frac{\tau+1}{2}\right)}{\eta(\tau)}, \quad \mathfrak{f}_{1}(\tau)=\frac{\eta\left(\frac{\tau}{2}\right)}{\eta(\tau)}, \quad \mathfrak{f}_{2}(\tau)=\sqrt{2} \frac{\eta(2 \tau)}{\eta(\tau)} .
$$

In fact

$$
a=\left|\mathfrak{f}_{1}((1+\sqrt{-2}) / 2)\right|=\left|\mathfrak{f}_{1}((-1+\sqrt{-2}) / 2)\right|,
$$

the latter equality following from (6).

By applying the modular substitution $\tau \rightarrow \tau+1$ to $\mathfrak{f}$, we find by the modular substitution rules for the Weber functions $([17] \S 34)$ that

$$
a=|\mathfrak{f}((1+\sqrt{-2}) / 2)| \text {. }
$$

Now we can use the elegant identity $([17] \S 34)$

$$
\mathfrak{f}(\tau) \mathfrak{f}_{1}(\tau) \mathfrak{f}_{2}(\tau)=\sqrt{2},
$$

which holds for all $\tau$ in the complex upper half plane. From (32) and (33) we have

$$
a^{2}\left|\mathfrak{f}_{2}((1+\sqrt{-2}) / 2)\right|=\sqrt{2} \text {. }
$$

Now we can use the identity $([17] \S 34)$

$$
\mathfrak{f}_{1}(\tau)=\frac{\sqrt{2}}{\mathfrak{f}_{2}(\tau / 2)}
$$

from which we obtain

$$
a^{2}=\left|\mathfrak{f}_{1}(1+\sqrt{-47})\right|=|\mathfrak{f}(\sqrt{-47})|
$$

making use of a final modular substitution $\tau \rightarrow \tau+1$ for the function $\mathfrak{f}_{1}$.

The value of $f(\sqrt{-47})$ is given in [Weber $\S 131(3)]$. He states that

$$
f(\sqrt{-47})=\sqrt{2} x \text {, where } x^{5}-x^{3}-2 x^{2}-2 x-1=0 .
$$

We note that Weber's calculation is not numerical, but provides a discrete method for evaluation involving a modular equation. Note that it appears that there may be a typographical error in the relation above $\S 131$ (1) in Weber, which ought to read $B=4 x+\frac{(-1)^{\frac{m+1}{8}}}{x^{2}}$.

Of course all the work here has been done for us by Weber who obtains the evaluation (38) using his theory of modular equations for the Weber functions. In the next section we apply similar techniques to the new modular equations of Weber type functions, found in [14], to obtain interesting evaluations for cases that can't be dealt with by any of our techniques so far. 


\section{Evaluations Via Modular Equations of Weber Type Functions}

In this section we will provide an eta evaluation at a point in the quadratic order of discrimnant $D=-2^{2} \cdot 71$, which has class number seven.

We will make use of a modular equation for the Weber-like functions of [14] of signature three. These functions are defined as follows

$$
\mathfrak{g}(\tau)=\sqrt{3} \frac{\eta(3 \tau)}{\eta(\tau)}, \quad \mathfrak{g}_{1}(\tau)=\frac{\eta\left(\frac{\tau}{3}\right)}{\eta(\tau)}, \quad \mathfrak{g}_{2}(\tau)=\frac{\eta\left(\frac{\tau+4}{3}\right)}{\eta(\tau)}, \quad \mathfrak{g}_{3}(\tau)=\zeta_{12}^{-1} \frac{\eta\left(\frac{\tau+8}{3}\right)}{\eta(\tau)}
$$

The following theorem from [14] describes the action of modular transformations on these functions

\section{Theorem 4.1.}

$$
\left(\begin{array}{c}
\mathfrak{g} \\
\mathfrak{g}_{1} \\
\mathfrak{g}_{2} \\
\mathfrak{g}_{3}
\end{array}\right) \circ S=\left(\begin{array}{c}
\zeta_{12} \mathfrak{g} \\
\zeta_{12}^{-1} \mathfrak{g}_{2} \\
\mathfrak{g}_{3} \\
\mathfrak{g}_{1}
\end{array}\right) \quad \text { and } \quad\left(\begin{array}{c}
\mathfrak{g} \\
\mathfrak{g}_{1} \\
\mathfrak{g}_{2} \\
\mathfrak{g}_{3}
\end{array}\right) \circ T=\left(\begin{array}{c}
\mathfrak{g}_{1} \\
\mathfrak{g} \\
\mathfrak{g}_{3} \\
\mathfrak{g}_{2}
\end{array}\right)
$$

where $S$ stands for the transformation $\tau \rightarrow \tau+1$ and $T$ for $\tau \rightarrow-1 / \tau$.

In order to obtain our eta evaluation we will need a modular equation of degree 71 for the function $\mathfrak{g}_{1}$. In section 5 of [14] the technique for constructing such a modular equation is described as follows. We abbreviate $u=\mathfrak{g}_{1}(\tau)$ and $v=\mathfrak{g}_{1}(71 \tau)$. We then define

$$
A=(u v)+3 /(u v) \quad \text { and } B=(u / v)^{6}+(v / u)^{6} .
$$

Now [14] then guarantees that if we find a polynomial relationship $F(A(q), B(q))$ between the $q$-series of $A$ and $B$ then $F(A, B)=0$ is a modular equation relating the two functions $A$ and $B$. We find such a polynomial $F$ relatively easily by making use of Pari [11] (see the Pari code below).

Once we have a modular equation, we substitute in a particular value of $\tau$. As it is, the modular equation is a polynomial relationship between $u=\mathfrak{g}_{1}(\tau)$ and $v=\mathfrak{g}_{1}(71 \tau)$. If we choose the value $\tau=2+-1 / \sqrt{-71}$ then $71 \tau=172+\sqrt{-71}$.

From the transformation laws (40) we easily see that these values become

$$
\begin{gathered}
u=\mathfrak{g}_{1}(2+-1 / \sqrt{-71})=\zeta_{12}^{-1} \mathfrak{g}_{2}(\sqrt{-71}) \\
v=\mathfrak{g}_{1}(172+\sqrt{-71})=\mathfrak{g}_{2}(\sqrt{-71}) .
\end{gathered}
$$

Thus the modular equation now only involves the value $\mathfrak{g}_{2}(\sqrt{-71})$.

In order to simplify the equation that we get, we write these values in terms of eta functions. In fact, if we let $t=\eta((\sqrt{-71}+1) / 3) / \eta(\sqrt{-71})$ then the functions $A$ and $B$ can be written as follows

$$
A=t^{2}+3 / t^{2} \quad \text { and } \quad B=-2 .
$$

Making these substitutions in our modular equation, we obtain a polynomial equation solely in $t$. Pari is able to factorize this polynomial of degree 140 and finally the square of our eta value, $x=t^{2}$, is found to have minimum polynomial

$$
\begin{aligned}
& (45) \quad x^{14}-48 x^{13}+806 x^{12}-5751 x^{11}+21673 x^{10}-45772 x^{9}+54296 x^{8}-57799 x^{7} \\
& +162888 x^{6}-411948 x^{5}+585171 x^{4}-465831 x^{3}+195858 x^{2}-34992 x+2187=0 .
\end{aligned}
$$

The Pari code for this process is as follows: 


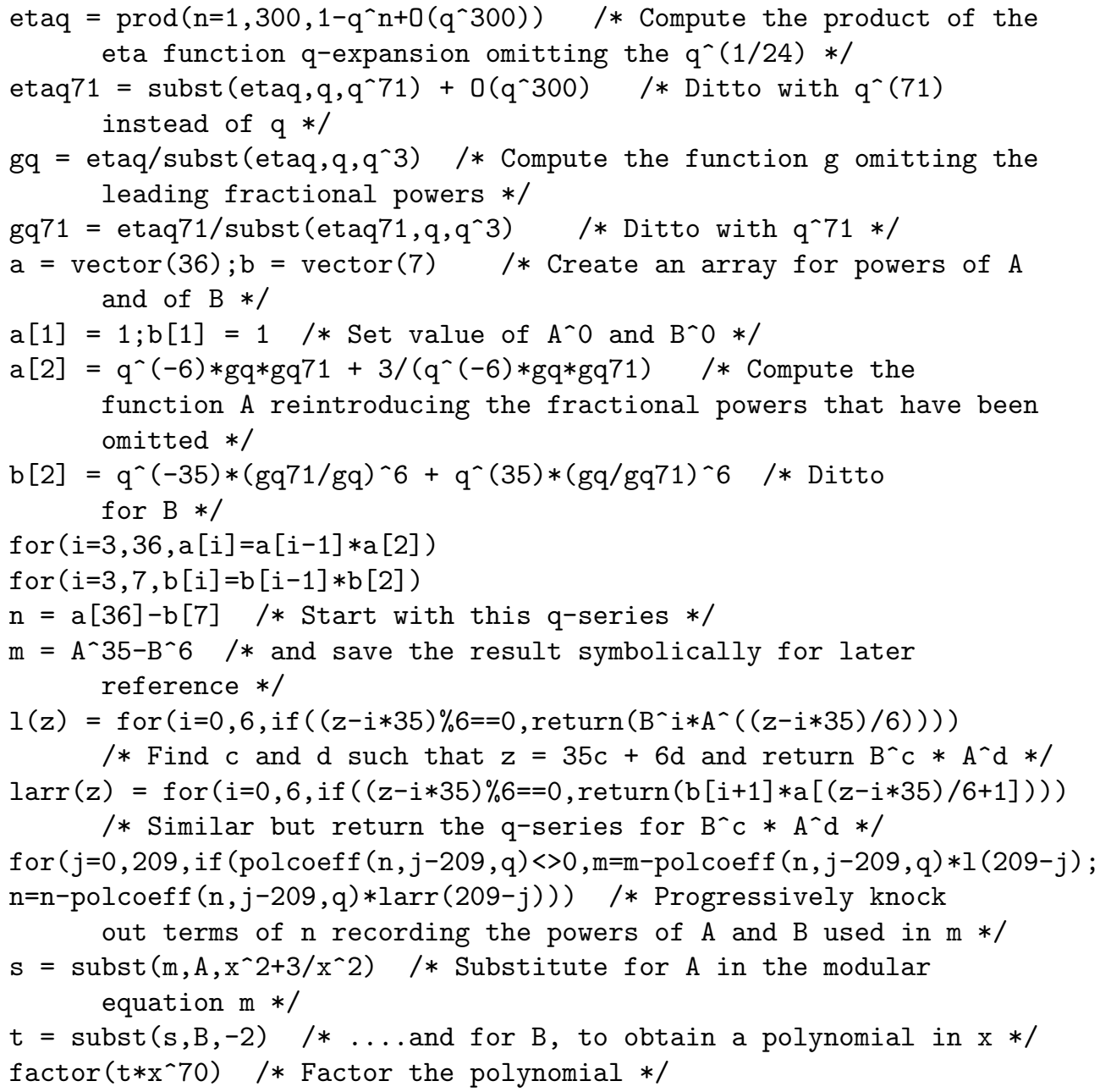

The modular equation of degree 71 that is obtained is as follows:

\footnotetext{
$-B^{6}+\left(45227 A^{5}+18133258 A^{4}+1004418327 A^{3}+15824008560 A^{2}+88322894388 A+157324900428\right) B^{5}$ 


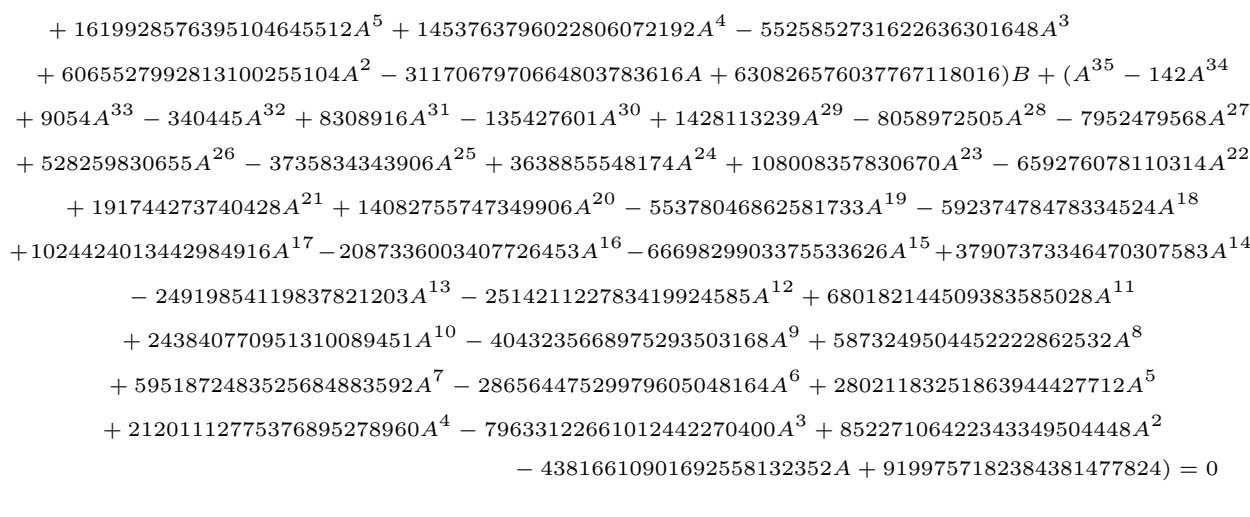

REFERENCES

[1] N. H. Abel Recherches sur les fonctions elliptiques J Reine Angew. Math. 2 (1828) 380-382

[2] See http://www.austms.org.au/AMSInfo/BHN/pastwinners.html

[3] Nan Y. Zhang, Kenneth S. Williams On the Epstein zeta function Tamkang J. Math. 26 (1995), 2, pp. 165-176.

[4] B. C. Berndt, S. Bhargava, and F. G. Garvan Ramanujan's Theories of Elliptic Functions to Alternative Bases Trans. AMS 347.11, (1995), 4163-4244.

[5] David A. Cox, Primes of the form $x^{2}+n y^{2}$. Fermat, class field theory and complex multiplication Wiley-Interscience, John Wiley \& Sons, Inc., New York, 1989.

[6] Andreas Enge and François Morain, Comparing invariants for class fields of imaginary quadratic fields Preprint.

[7] Alfred J. van der Poorten, Robin Chapman Binary Quadratic Forms and the Eta Function - Proceedings of the Millenial Conference (2001) (Urbana Champagn)

[8] James G. Huard, Pierre Kaplan, Kenneth S. Williams The Chowla-Selberg Formula for Genera Acta. Arith. LXXIII.3 (1995) pp 271-301.

[9] S. Chowla, A. Selberg On Epstein's zeta-function J. Reine Agnew. Math. 227 (1967), 86-110

[10] Farshid Hajir and Fernando Rodriguez-Villegas Explicit Elliptic Units I Duke Math. J. 90, (1997) 495-521

[11] PARI-GP. See http://pari.math.u-bordeaux.fr.

[12] Alice Gee and Peter Stevenhagen, Generating Class Fields Using Shimura Reciprocity, in ANTS III, LNCS 1423 (1998), 441-453.

[13] William Hart, PhD Thesis. Macquarie University, Sydney, to be completed in early 2004.

[14] William Hart, Schlaefli Modular Equations for Generalized Weber Functions Preprint.

[15] Hans Rademacher and Emil Grosswald, Dedekind Sums, Carus Math. Monographs, 16, MAA, Washington, D.C., 1972.

[16] Bruno Schoeneberg, Elliptic Modular Functions, Grundl. der math. Wiss. 203, SpringerVerlag, New York-Heidelberg, 1974.

[17] Heinrich Weber, Lehrbuch der Algebra, Dritter Band, Third Edition. Chelsea N.Y.

[18] Alfred J. van der Poorten and Kenneth S. Williams Values of the Dedekind Eta Function at Quadratic Irrationalities Can. J. Math. vol. 51 (1) (1999) pp. 176-224 + Corr. vol. 53 (2) (2001) pp. 434-448.

[19] Masanobu Kaneko, A generalization of the Chowla-Selberg formula and the zeta functions of quadratic orders Proc. Japan Acad. Ser. A Math. Sci. 66 (1990), no. 7, 201-203 (MR91i:11166).

[20] Y. Nakkajima and Y. Taguchi, A generalization of the Chowla-Selberg formula, J. Reine Angew. Math. 419 (1991), 119-124 (MR92g:11113).

[21] I. J. Zucker, M. M. Robertson Exact Values for some Two-dimensional Lattice Sums J. Phys. A Vol. 8, No. 6 (1975) pp 874-881.

[22] I. J. Zucker, M. M. Robertson Some Properties of Dirichlet L-series J. Phys. A Vol. 9, No. 8 (1976) pp 1207-1214.

[23] I. J. Zucker, M. M. Robertson A Systematic Approach to the Evaluation of $\sum_{(m, n \neq 0,0)}\left(a m^{2}+b m n+c n^{2}\right)^{-s}$ J. Phys. A Vol. 9, No. 8 (1976) pp 1215-1225.

[24] I. J. Zucker, M. M. Robertson Further Aspects of the Evaluation of $\sum_{(m, n \neq 0,0)}\left(a m^{2}+\right.$ $\left.b m n+c n^{2}\right)^{-s}$ Math. Proc. Camb. Phil. Soc. (1984), 95, pp 5-13.

E-mail address: wb_hart@maths.mq.edu.au 